\title{
Current Urology
}

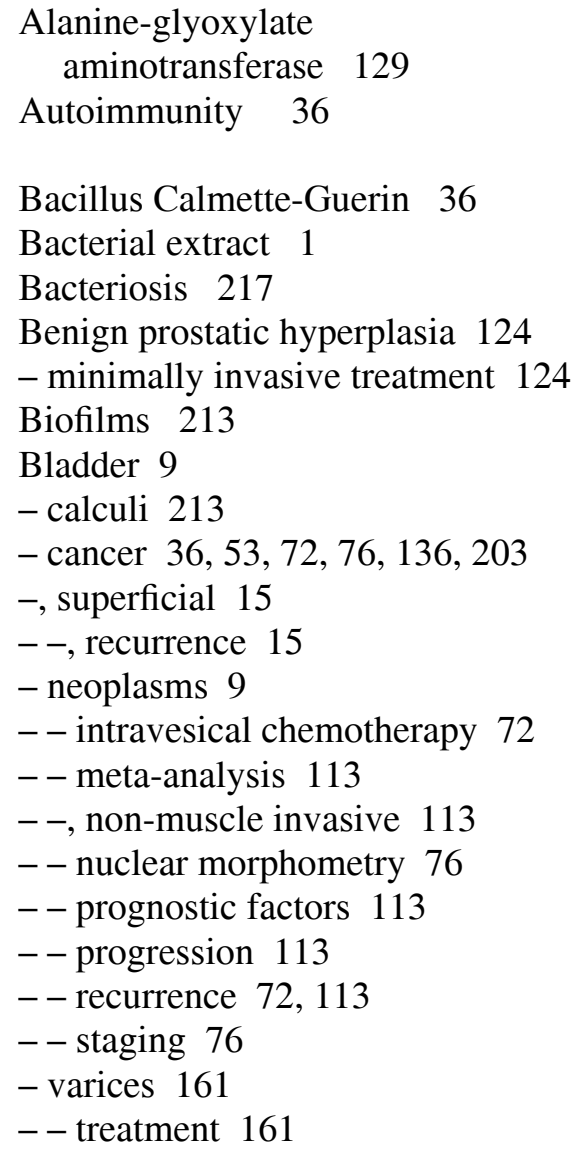

Calcium oxalate 129

Cell cycle 136

Cellular proliferation 136

Chronic inflammation 174

Computerized tomography 169

Conservative therapy 110

Continent diversion 199

Continent stoma 199

Corpus cavernosum 217

- abscess 217

Cyanoacrylic glue 194

Cystectomy 9

- postoperative complications 9

-, radical 203

-- , retrograde 203
Denonvillier's fascia 141

Detrusor 15, 57

- contractility 19

- overactivity 57

Electromotive drug administration 15

Endometriosis 110

Erectile dysfunction 217

- etiology 217

Erection 146

Erosion 82

Escherichia coli 1

Ethylene glycol 129

Extracorporeal shockwave therapy 169

Extrusion 82

Female to male transsexual 146

Foreign body 156

Frozen section examination 158

Gender reassignment surgery 146

Genital renal ear syndrome 103

Germ cell tumor 207

Granulocyte colony-stimulating factor 53

Hemangiopericytoma 41

Hematuria 161

HIV infection 158

Ileal neobladder 203

Ileal neourethra 203

Immunostimulation 1

Immunotherapy 1

Indwelling catheter 185

Infection 217

Inositol hexaphosphate 136

Interferon alpha $2 b 36$

Intermittent catheterization 156

Interstitial cystitis 9

Intraurethral catheter 185
Kidney 106

- cyst 106

- hemangioma 106

- neoplasm 106

Laparoscopy 194

Local anesthesia 15

Male genital diseases 217

Mass spectroscopy 129

Mayer-Rokitansky-Küster-Hauser syndrome 103

Mesh 82

Micturition 146

Modified mitrofanoff 199

Monarc transobturator sling operation 49

Mullerian aplasia 103

Nephrectomy 100, 106

Nephrolithiasis 156

Nephropexy 194

Nephroptosis 194

Nephroureterectomy 100

OM-89 1

Orchiectomy 190, 210

Paratesticular mass 158

Parathyroid-hormone-related protein 53

Penile diseases 217

Penile prosthesis 146

Percutaneous nephrostomy 174

Periodontopathy 217

Phalloplasty 146

Polypoid tumor 110

Posterior median raphe 141

Precutaneous nephrostomy lithotripsy 67

Pressure-flow study 19

Primitive neuroectodermal tumor 207 
Prolapse 82

Prostate 87, 94, 190

- adenocarcinoma 94

- cancer 87, 190

- - biochemical recurrence 62

- - detection 29

- - laparoscopy 94

- - - anastomotic suture 94

- - - suture device 94

- - radical transurethral resection

87

- - radical treatment 87

- - T1c 62

prostatectomy $29,62,94,141$

- , radical 62,94

-- , retropubic 141

-, suprapubic 29

- surgical margin 62

Prostate specific antigen 29

Pyeloplasty 174

Renal agenesis 103

Renal cell carcinoma 164

- laparoscopy 164

- neoplasm seeding 164

- recurrence 164

Renal neoplasm 41

Reproducibility 19
Retroperitoneal tumor 41

Retroperitoneoscopy 94

Retrotrigonal tissue 141

Scrotum 44

- mass 44

Slings 82

Spinal cord injuries 213

Squamous cell carcinoma 67

Staghorn stone 67

Stone disease 169

Streptococcus 217

Tension-free vaginal tape 179

- obturator 179

Teratoma 207

Testicular

- cancer 44

- mass 44, 210

- sarcoma 44

- sparing 158

- torsion 158

- tumor 44

Transitional cell carcinoma 67

Transurethral resection of prostate 124

Two-dimensional electrophoresis 129
Ureter

-, circumcaval 100

-, retrocaval 100

Ureteral obstruction 110

Ureterocystoneostomie 110

Urethral resistance 19

Urinary catheterization 213

Urinary diversion 9

Urinary incontinence 57

- diagnosis 57

-, stress 49, 179

- -, ambulatory treatment 49

- -, mathematical model 179

,-- recurrent 49

- - uroflowmetry 179

- urodynamics 57

Urinary tract infection $1,185,213$

Urogenital sinus anomaly 103

Urolithiasis 129

Urothelial carcinoma 100

Uro-Vaxom 1 